\title{
La responsabilidad social en la Biblioteca Universitaria a través del Plan en Fomento del Libro, la Lectura y la Escritura
}

Social responsibility at the Universitary Library through the Plan on Promotion of the Book, Reading and Writing

Ricardo Chamorro

Subdirector de Biblioteca y Archivo.

Universidad de Cádiz

ricardo.chamorro@uca.es

\section{Resumen}

Cuando en la universidad se detecta la necesidad de incluir la responsabilidad social en sus ámbitos de actuación para cumplir con su misión y desarrollar acciones que potencien la formación integral en valores de sus miembros, así como impulsar actividades encaminadas a promover, realizar y obtener evidencias del papel abierto de la universidad, como parte activa de la sociedad y su entorno, desde la biblioteca de la Universidad de Cádiz se promueve un plan para desarrollar todos estos aspectos de una forma más específica por lo que se pone manos a la obra y de todo ello nace el "Plan Universitario en Fomento del Libro, la Lectura y la Escritura". A través de este, la biblioteca universitaria con la colaboración del Servicio de Publicaciones de la UCA ha venido experimentando un nuevo concepto y modelo de los servicios universitarios con el que, además de dar soporte al aprendizaje, la docencia, la investigación y la innovación docente, se ha llevado a cabo un conjunto de programas y actividades orientadas a cubrir otras necesidades académicas y del entorno social de la Universidad.

\begin{abstract}
The "University Plan on promotion of the book, reading and writing" is promoted from the library of the University of Cádiz after detecting the need of including the social responsibility in their areas of performance in order to achieve their objectives and develop actions that boost it training integral in values of their members, as well as boost activities aimed to promote, perform and get evidence of the paper open of the University, as part active of the society and its environment. Based on this plan, the University Library with the collaboration of the publications service of the UCA has been experiencing a new concept and model of University services which, in addition to support learning, teaching, research, and teaching innovation, has carried out a series of programs and activities to meet other academic needs and the social environment of the University.
\end{abstract}

Palabras clave: Biblioteca, Universidad de Cádiz, Plan Universitario en Fomento del Libro, la Lectura y la Escritura

Keywords: Library, University of Cadix, University plan on promotion of books, reading and writing 


\section{Introducción}

La Universidad de Cádiz (en adelante, UCA) implantó su primer código ético de conducta en el año 2005, cuando el Claustro Universitario aprobó en su sesión de 29 de noviembre de ese mismo año el "Código Ético de la Universidad de Cádiz", también conocido por el nombre de "Código Peñalver".

Mariano Peñalver Simó (1930-2005) había sido elegido Rector en las primeras elecciones que se celebraron en la Universidad de Cádiz, cargo que ocupó desde febrero de 1984 hasta junio de 1986. Durante su etapa se aprobaron los primeros Estatutos de la UCA, fruto de un intenso trabajo. En el "Código Peñalver", que nos dejó como legado poco antes de su fallecimiento, se hace gran hincapié en su "no obligatoriedad" y en que su fuerza se basa en la voluntariedad, como cita en el punto cinco de las consideraciones preliminares:

La fuerza de la conducta ética depende justamente de su no obligatoriedad jurídica o administrativa. Su incumplimiento no desencadenará, por tanto, ninguna sanción externa. El propio sujeto ético-moral auto-evalúa su propia conducta. Una conducta, que quizá puede llegar a suscitar la estima, la admiración o el reconocimiento de los otros miembros de la institución universitaria en la que vive (Peñalver, 2005, p.3).

Otro de los aspectos destacables de las consideraciones preliminares, donde se concentra de forma resumida todo un abanico de valores y de propuestas, es el respeto que se muestra al reconocimiento de la diversidad, el respeto que se quiere dar al medio ambiente y el sentimiento de pertenecer a un proyecto. Todos estos aspectos prefiguran lo que luego será el concepto de responsabilidad social.

La Universidad de Cádiz aspira a ser creativa, dinámica, innovadora, emprendedora, proactiva, con idea de equipo y vocación de servicio público e implicación en un proyecto común (Peñalver, 2005, p. 4).

El "Código Peñalver" además de las consideraciones preliminares contiene un apartado dedicado a los "Cargos Académicos", otro dedicado al "Personal Docente e Investigador", otro al "Alumnado" y otro al "Personal de Administración y Servicios". En este apartado se dice que:

Cada miembro del Personal de Administración y Servicios debe sentirse éticamente comprometido en todo momento no sólo a cumplir con sus funciones administrativas o técnicas sino a participar, en el campo de sus competencias profesionales, en el desarrollo de la institución universitaria a la que pertenece (Peñalver, 2005, p. 4).

Partiendo de estas premisas y con la vista puesta en el nuevo Espacio Europeo de Educación Superior (en adelante, EEES) en nuestra universidad se detecta la necesidad de incluir la responsabilidad social en sus ámbitos de actuación para cumplir con su misión y desarrollar acciones que potencien la formación integral de sus miembros en los valores de la solidaridad, el respeto, la tolerancia, la libertad, así como impulsar actividades encaminadas a promover, realizar y obtener evidencias del papel abierto de la universidad, como parte activa de la sociedad y su entorno. 
Y es así como, desde la biblioteca de la Universidad de Cádiz, se va haciendo cada vez más consciente de la necesidad de desarrollar todos estos aspectos de una forma más específica por lo que se pone manos a la obra. De todo ello nace en el año 2012 el Plan Universitario en Fomento del Libro, la Lectura y la Escritura. A través de este, la biblioteca universitaria con la colaboración del Servicio de Publicaciones de la UCA ha venido experimentando un proceso de adaptación orientado hacia un nuevo concepto y modelo de los servicios universitarios con el que, además de dar soporte al aprendizaje, la docencia, la investigación y la innovación docente, ha llevado a cabo un conjunto de programas y actividades orientadas a cubrir otras necesidades académicas y del entorno social de la Universidad.

\section{Descripción del Plan}

El Plan Universitario en Fomento del Libro, la Lectura y la Escritura forma parte de la planificación estratégica de la biblioteca universitaria en colaboración con el Servicio de Publicaciones de la UCA. Gracias al conjunto de programas que lo componen se desarrolla una serie de actividades dirigidas a toda la comunidad universitaria y a la sociedad en general, con las que se pretende explorar y elevar los hábitos lectores, así como aumentar la comprensión lectora. En definitiva se busca potenciar todos los esfuerzos con el fin de facilitar el acceso a las lecturas; reconocer y promocionar al autor y su obra; estimular la creación literaria, generar experiencias en lecturas y escrituras, así como preservar y poner en valor el patrimonio bibliográfico.

Bajo esta idea y ámbitos diversos, de manera coordinada y desde una mirada integral y global, la Biblioteca de la UCA ha adaptado y vinculado su plan operativo al Plan Estratégico de la Universidad de Cádiz (en adelante, PEUCA), siempre con la vista puesta en el EEES, en el que se impulsan una serie de mejoras en los sistemas educativos de los estados de la Unión Europea.

De acuerdo con los objetivos recogidos en el PEUCA y como desarrollo de su planificación estratégica - dirigidas especialmente a todo el alumnado, con independencia de su edad y de las enseñanzas que curse, y resto de la comunidad universitaria (PDI y PAS), así como a la sociedad en general, a través del Plan Universitario en Fomento del Libro, la Lectura y la Escritura, y el conjunto de programas y actividades - se desarrolla un amplio despliegue en las siguientes líneas de acción:

$\checkmark \quad 5.3$ Contribuir al desarrollo social y humanístico con el conocimiento generado en la Universidad.

$\checkmark \quad 8.1$ Impulsar la participación del alumnado y de nuestros egresados en la Universidad.

$\checkmark \quad$ 9.1 Reforzar la formación integral de los estudiantes.

Su organización, coordinación y desarrollo, se lleva a cabo a través de la biblioteca universitaria desde cuya dirección, junto con el Grupo de Responsabilidad Social del Área de Biblioteca y Archivo y el Servicio de Publicaciones, se lidera una serie de programas y actividades como proceso de adaptación orientado hacia un nuevo concepto y modelo de los servicios universitarios. 


\section{Objetivos y programas del plan}

El principal objetivo del Plan Universitario en Fomento del Libro, la Lectura y la Escritura es el de promocionar y dinamizar la Biblioteca de la UCA, su fondo bibliográfico y servicios como un espacio para el fomento del trabajo literario y de investigación de la comunidad universitaria y la sociedad en general, que se vertebran en las siguientes líneas básicas de actuación:

- Reconocimiento y promoción del trabajo literario y de investigación realizado por miembros de la comunidad universitaria de la UCA y de su entorno social.

- Promoción, dinamización y puesta en valor del libro, a través de los servicios y entidades que lo generan, mantienen y custodian.

- Impulsar una conciencia social sobre la importancia del libro, la lectura y la escritura.

- Colaborar en la organización y acometer la realización de actividades de promoción del libro, y la lectura y la escritura en diferentes ámbitos.

A través del Plan Universitario en Fomento del Libro, la Lectura y la Escritura, con el fin de impulsar de forma coordinada en los cuatro campus universitarios, y por ende en los municipios donde se ubican, se está realizando un conjunto de actividades para incrementar los hábitos lectores y aumentar la comprensión lectora. Estas actividades pretenden, buscando potenciar todos los esfuerzos para de facilitar el acceso a la lectura; el reconocimiento y promoción al autor y su obra; la estimulación para la creación literaria, generando experiencias en lecturas y escrituras, así como la preservación y puesta en valor del patrimonio bibliográfico mediante acciones de dinamización, divulgación y formativas.

Para el cumplimiento de estos objetivos, se han puesto en marcha los siguientes programas y actividades:

\section{Certamen Literario de Narrativa Breve}

Con este certamen se busca asentar un espacio de expresión y creatividad para los estudiantes de la Universidad de Cádiz. Los relatos premiados son recopilados en un libro editado con el sello editorial del Servicio de Publicaciones de la UCA, formará parte de la colección "Libro de Bienvenida" que a su vez es otro de los programas que forman parte del plan y con él se obsequiará a todos los alumnos y alumnas de nuevo ingreso.

Cabe resaltar la colaboración del Consejo Social, así como su implicación en el Jurado del Certamen que cuenta con la participación de personalidades de reconocido prestigio académico (escritores, docentes y personal de administración y servicios).

\section{Libro de Bienvenida}

El Libro de Bienvenida es el resultado final del Certamen Literario de Narrativa Breve de la Biblioteca Universitaria de Cádiz. Cada año, las obras premiadas se recopilan en 
un libro editado con el sello de la editorial UCA y que pasa a formar parte de la colección "Libro de Bienvenida" con el que se obsequia al alumnado de nuevo ingreso para promover el hábito a la lectura y como simbología de la relación que, a partir de su entrada en la Universidad, van a mantener en su vida académica y profesional, con el libro y la biblioteca.

\section{Se ha escrito un libro por...}

A través de este programa se le ofrece a aquellas personas, vinculadas con la comunidad universitaria y su entorno social, que han escrito y editado algún libro la oportunidad de presentarlo dentro de la propia institución, difundirlo y hacerlo público para la sociedad gaditana en general, así como su inserción en el fondo bibliográfico de la Biblioteca de la UCA, pues, una vez que ha finalizado el acto de presentación, el autor firma un ejemplar que pasa a formar parte de la colección "Se ha escrito un libro por...", del catálogo de la Biblioteca de la UCA.

\section{Los Libros Olvidados}

Con este programa se pretende rescatar y poner en valor las obras de la colección histórica de la Universidad de Cádiz y darlas a conocer ante su comunidad y la sociedad en general, mostrándole la importancia y transcendencia de su contenido en los estudios, enseñanza e investigación de su época. Para su realización desde el Grupo de trabajo de la colección histórica se anima a los miembros de la comunidad universitaria a releer o leer por primera vez obras que se publicaron hace tiempo. Rescatar obras en el olvido, libros raros, curiosos. Redescubrir historias, investigaciones, imágenes, documentos, poemas, escritos.... Y valorar su importancia en la formación académica y la investigación de la época, así como su propia vigencia.

Mediante exposiciones y presentaciones públicas de las obras, por una parte, y la restauración de obras de la colección histórica, por otra, se difunde, recupera y pone en valor este importante patrimonio bibliográfico de la Universidad de Cádiz.

\section{Libros Libres - Bookcrossing}

Desde el Vicerrectorado de Alumnado de la UCA se puso en marcha en el año 2008 esta iniciativa para fomentar la lectura y difundir entre la comunidad Universitaria un espíritu de compartir recursos. Consiste en dejar libros en lugares públicos para que los recojan otros lectores, que después harán lo mismo. La idea es dejar viajar libremente a los libros para que puedan ser encontrados por otras personas.

Es así como, a partir del año 2012, desde la biblioteca de la UCA el Servicio de Publicaciones de la UCA hemos cogido y relanzado esta iniciativa bajo la denominación de Libros Libres - Bookcrossing y con el fin de ayudar a fomentar la lectura entre el alumnado universitario. En definitiva, se trata de fomentar la cultura del disfrute del libro por encima de la posesión. 


\section{Este Libro es para Ti}

Este programa contempla la donación de libros no ingresados o retirados del fondo bibliográfico de la Biblioteca de la UCA.

Con este programa se persigue la concienciación entre los alumnos, profesores y personal de la UCA de la utilidad permanente de los libros. Por una parte, la Biblioteca y el Servicio de Publicaciones de la UCA ponen a disposición de la comunidad universitaria aquellos libros retirados de sus fondos, así como los provenientes de donaciones no ingresados en el fondo bibliográfico de la Biblioteca de la UCA, de conformidad a la normativa vigente de expurgo y donaciones.

\section{Semana Universitaria del Libro}

Día Internacional del Libro es una conmemoración que se estableció por primera vez en 1995 a nivel internacional por la UNESCO con el objetivo de fomentar la lectura, la industria editorial y la protección de la propiedad intelectual por medio del derecho de autor, así como rendir un homenaje mundial al libro y sus autores, y alentar a todos, en particular a los más jóvenes, a descubrir el placer de la lectura y respetar la irreemplazable contribución de los creadores al progreso social y cultural. El día 23 de abril fue elegido como Día Internacional del Libro, por la coincidencia con el fallecimiento de Miguel de Cervantes, William Shakespeare y el Inca Garcilaso de la Vega en la misma fecha en el año 1616.

Con motivo de esta efeméride, y como colofón al plan en fomento del libro, la lectura y la escritura, desde la biblioteca de la UCA se organizan y promueven en los cuatro campus universitarios una serie de actividades, que cuentan también con el apoyo del Consejo Social, centros, departamentos universitarios, otros servicios de la propia universidad, así como diversas asociaciones y colectivos sociales

\section{Alcance, resultados e implantación del Plan}

Dada la diversidad de programas que lo forman, de agentes que participan e intervienen, hasta el momento actual y desde su puesta en marcha a comienzos del curso académico 2012-2013, el Plan Universitario en Fomento del Libro, la Lectura y la Escritura ha supuesto, sobre todo, un cambio en la función y cometido de la biblioteca universitaria $\mathrm{y}$ el servicio de publicaciones que han pasado a tener una mayor visibilidad en su entorno social a través de las instituciones y el conjunto asociativo que lo representan.

Esta política de servicios y de apertura, todavía en desarrollo, así como las unidades administrativas que lo despliegan, además del Sello de Excelencia Europea 500+, otorgado al Área de Biblioteca y Archivo de la UCA por el Club de Excelencia en Gestión y el Reconocimiento de la Excelencia 5 estrellas, concedido por la Fundación Europea para la Gestión de Calidad, ha obtenido una serie de resultados importantes, multitud de agradecimientos e incluso reconocimientos locales, nacionales e internacionales.

A continuación, se relacionan alguno de estos resultados y reconocimientos: 
$\checkmark$ Presentación en los Cursos de Verano de la Universidad de Cádiz dentro del II y III Seminario Estival de la Red Internacional de Universidades Lectoras que tiene como objetivos el análisis y la valoración de las iniciativas que en política de lectura se llevan a cabo en distintos contextos internacionales, nacionales y autonómicos a través de la articulación de programas y proyectos de muy diferente índole.

$\checkmark$ Premio Mojarrita de Plata 2014, concedido por la Asociación Amigos de Fernando Quiñones.

$\checkmark$ Premio 3M a la Mejor Práctica de Calidad.

$\checkmark$ Bandera de Andalucía 2014 en la modalidad de Innovación e Investigación Científica, reconocimiento concedido por la Junta de Andalucía.

$\checkmark$ Implantación de la "huella solidaria" en el libro de bienvenida, editado por el Servicio de Publicaciones a partir del segundo semestre del año 2014. Con este distintivo el Servicio de Publicaciones manifiesta su compromiso de donar parte de los ingresos generados por la venta de libros a un fondo social.

$\checkmark$ Compromiso ambiental y Ecoedición. Participación en el proyecto Ecoedición, coordinado por la Consejería de Medio Ambiente y Ordenación del Territorio de la Junta de Andalucía y cofinanciado por la Unión Europea en el marco del Programa LIFE+. Todos los libros de bienvenida editados a través de la Editorial UCA muestran el distintivo de ecoedición en el que figuran los datos de compromiso ambiental y catalogan a ese libro como "libro verde".

Sin duda, el Plan Universitario en Fomento del Libro, la Lectura y la Escritura, ha tenido un impacto clave en el papel de la Biblioteca de la UCA y la actividad que este servicio venía desempeñando para la comunidad universitaria, pero sobre todo ha desempeñado un factor fundamental como indicador para la calidad de sus cometidos como servicio y su compromiso con la responsabilidad social.

Por parte de los responsables de su organización y coordinación, cabe resaltar como más destacable la apertura de los servicios universitarios a su entorno social y la relación con las entidades y tejido asociativo que lo representa, habiéndose realizado acuerdos con algunos de ellos y realizado actos que han dado respuesta a una antigua demanda de muchos ciudadanos y colectivos que reclaman una mayor utilidad de la Universidad como institución al servicio de la sociedad gaditana y su tejido económico y cultural.

Gracias a este Plan, se han abierto las puertas de la universidad a antiguos alumnos, profesionales, empresas y asociaciones, pero sobre todo está llevándose a cabo una permanente llamada de atención y campaña de concienciación entre los miembros de la propia comunidad universitaria sobre cuál debe ser su cometido y sentido para con su entorno social y cultural.

Uno de los valores diferenciales del Plan Universitario en Fomento del Libro, la Lectura y la Escritura, es el bajo coste económico para llevarlo a cabo, que se basa en la idoneidad de recursos materiales y humanos unidos para la consecución de los objetivos propuestos. Por una parte, habiéndose logrado el necesario equilibrio y complementariedad entre los integrantes profesionales para su elaboración y, por otra, entre los participantes en cada uno de los programas y actividades con un reparto de tareas y ponderación de la participación pública y privada en su ejecución. 
En la realización de este proyecto, además del Vicerrectorado de Responsabilidad Social, Extensión Cultural y Servicios de la UCA, así como las direcciones y profesionales de las unidades universitarias que lo lideran, participan todos los estamentos que integran la comunidad universitaria (Alumnado, Personal Docente y de Investigación, Personal de Administración y Servicios) junto con asociaciones y colectivos sociales, empresas y entidades públicas del entorno.

Finalmente, el ámbito territorial que abarca en su desarrollo el Plan Universitario en Fomento del Libro, la Lectura y la Escritura, viene marcado por la propia peculiaridad de la Universidad de Cádiz cuya estructura se encuentra fuertemente vinculada al territorio en el que desarrolla su actividad en cuatro campus universitarios, en una provincia singular por las características de sus comarcas y por la distribución de población en torno a grandes núcleos urbanos.

\section{Referencias Bibliográficas}

Código deontológico del Área de Biblioteca y Archivo de la Universidad de Cádiz. 2008. https://goo.gl/LdOa1O

Grupo Estratégico para el Estudio del Impacto socioeconómico de las Bibliotecas en la Sociedad. El impacto económico y social de las bibliotecas: Informe de aproximación, Consejo de Cooperación Bibliotecaria, 2013.

Herrera-Morillas, J. L., Castillo-Díaz, A., Pérez-Pulido, M. (2014). Responsabilidad social y sostenibilidad en las bibliotecas universitarias españolas. El profesional de la información, 2014, marzo-abril, 23, n. 2.

Peñalver Simó, M. Código ético de la Universidad de Cádiz, aprobado por acuerdo del Claustro Universitario, adoptado en su sesión del 29 de noviembre de 2005. https://goo.gl/IGb6pD

Torres Santo Domingo, M. (2005). La función social de las bibliotecas universitarias. Boletín de la Asociación Andaluza de Bibliotecarios, 80, 43-70. 